\title{
Hematoma extradural na infância
}

\author{
Carlos Umberto Pereira*, João Domingos Barbosa Carneiro Leão**, Alvino Dutra da \\ Silva**, Gustavo Cabral Duarte*** \\ Serviço de Neurocirurgia do Hospital João Alves Filho. Aracaju, SE
}

\section{RESUMO}

Neste trabalho, os autores apresentam sua experiência em 44 casos de hematoma extradural na infância. Houve predomínio do sexo masculino com 33 casos. As principais causas foram queda acidental e acidentes de trânsito. O quadro clínico principal foi cefaléia, seguida de vômitos, hemiparesia, anisocoria, convulsões e, em 2 casos, anemia. A localização mais comum foi a região temporal, seguida da fossa posterior e, em apenas 1 caso, bilateral. $O$ exame de raios $X$ simples de crânio demonstrou traço de fratura em 32 casos e a tomografia computadorizada foi positiva em todos pacientes. Lesões intradurais associadas estavam presentes em 8 casos. 0 tratamento cirúrgico foi realizado em 40 casos e o conservador, em 4 . 0 resultado do tratamento foi excelente em 36 casos, regular em 4 e o óbito ocorreu em 4.

Os casos que tiveram o diagnóstico precoce apresentavam bom nível de consciência na admissão, em que o tratamento cirúrgico realizado precocemente e na ausência de lesões intradurais associadas cursaram com bom prognóstico.

\section{PALAVRA-CHAVE}

Hematoma extradural na infância.

\section{ABSTRACT}

\section{Epidural hematoma in children}

The authors present their experience in the treatment of 44 cases of epidural hematoma in infants. There was a predominance in males (33:11 male/female ratio). Accidental falls and traffic accidents were the most frequent causes of trauma. The main clinical manifestation were headaches, followed by vomiting, hemiparesis, anisocoria and seizures; anemia were found in two cases. The most frequent place for these hematomas was the temporal region, followed by the posterior fossa; in only one case it was bilateral. Signs of bone fracture were found in the plain X-ray in 32 cases and the CT scans revealed the lesion in all patients. Intradural lesions were associated in eight cases. Surgical treatment was performed in 40 patients and four patients were conservatively treated. The results of the treatment were excellent in 36 cases, fair in 4 and 4 patients died. The main prognostic factors for these patients were their alertness at the admission, early surgical treatment and absence of associated intradural lesions.

\section{KEYWORD}

Infantile epidural hematoma.

\section{Introdução}

O hematoma extradural (HED) é considerado uma complicação rara de traumatismo cranioencefálico na infância ${ }^{35}$. Suas causas e características clínicas são diferentes das dos adultos jovens ${ }^{4,10,15}$. O HED na era da tomografia computadorizada (TC) tem apresentado um prognóstico bom ${ }^{7,34}$. Nessa patologia, tem sido amplamente documentado que a ausência de diag- nóstico precoce e a presença de lesões intradurais associadas são fatores que contribuem para elevada morbidade e mortalidade ${ }^{20,27}$. No presente trabalho, os autores apresentam sua experiência em 44 casos de HED na infância e discutem causas, quadro neurológico, exames complementares, tratamento e resultados.

\footnotetext{
* Prof. Adjunto Doutor do Departamento de Medicina da Universidade Federal de Sergipe. Neurocirurgião do Serviço de Neurocirurgia do Hospital João Alves Filho, Aracaju, Sergipe.

** Neurocirurgião do Serviço de Neurocirurgia do Hospital João Alves Filho, Aracaju, Sergipe.

*** Acadêmico de Medicina da Universidade Federal de Sergipe, Aracaju, Sergipe.
} 


\section{Casuística e método}

Durante o período de janeiro de 1996 a maio de 2001, 44 pacientes portadores de hematoma extradural com idades entre 4 meses e 15 anos foram tratados no Serviço de Neurocirurgia do Hospital João Alves Filho (Aracaju, $\mathrm{SE}$ ). Nesse mesmo período, 28 crianças foram tratadas de HED nesse mesmo hospital por outras equipes neurocirúrgicas. Nossos pacientes foram estudados retrospectivamente quanto a idade, sexo, causas do hematoma, localização do hematoma, exame neurológico, exames complementares, tratamento e resultados.

\section{Resultados}

Nesta casuística, 33 crianças eram do sexo masculino e 11 eram do feminino (Gráfico 1). Quanto à idade, 9 pacientes tinham idade abaixo de 5 anos; 13 , entre 5 e 10 anos; 22, entre 10 e 15 anos (Gráfico 2). As causas do HED foram: queda acidental em 18 casos, acidentes de trânsito em 16, agressão física em 6, pós-craniotomia em 1 e desconhecida em 3 casos (Gráfico 3). As queixas e os sinais predominantes foram: cefaléia (32 casos), vômitos ( 24 casos), hemiparesia (19 casos), anisocoria (12 casos), crises convulsivas ( 11 casos) e anemia ( 2 casos) (Gráfico 4 ). A localização do hematoma foi temporal em 14 casos, fossa posterior em 10 , frontal em 9 , temporoparietal em 5 , parietal em 5, occipital em 2 e bilateral em 1 caso (Gráfico 5). O exame de raios $\mathrm{X}$ simples de crânio demonstrou traço de fratura em 32 pacientes e a tomografia computadorizada foi positiva em todos os casos (Gráfico 6). Lesões intracranianas associadas estiveram presente em 8 casos. Quarenta pacientes foram submetidos a tratamento cirúrgico e 4, ao conservador (Gráfico 7). O resultado do tratamento foi excelente em 36 casos, regular em 4 casos e o óbito ocorreu em 4 casos (Gráfico 8).

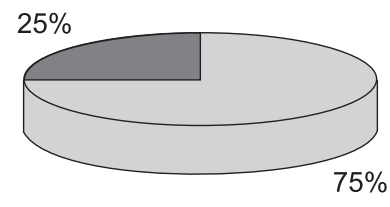

$\square$ Masculino $\quad \square$ Feminino

Gráfico 1 - Sexo.

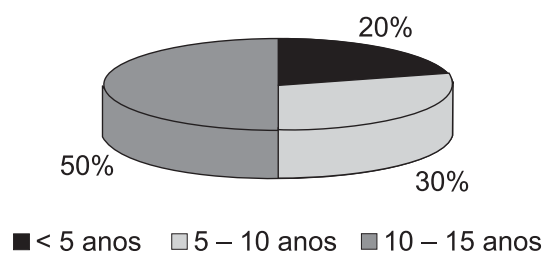

Gráfico 2 - Idade.

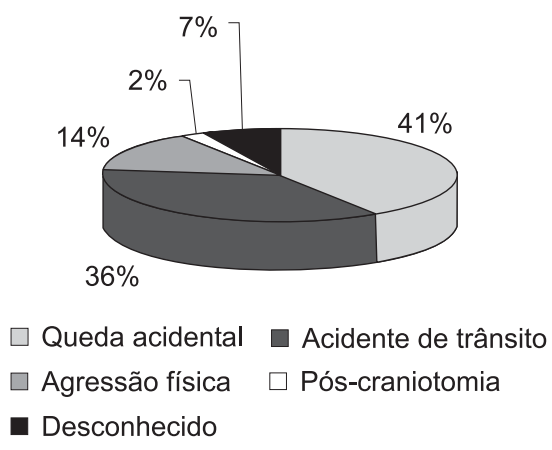

Gráfico 3 - Causas de HED.
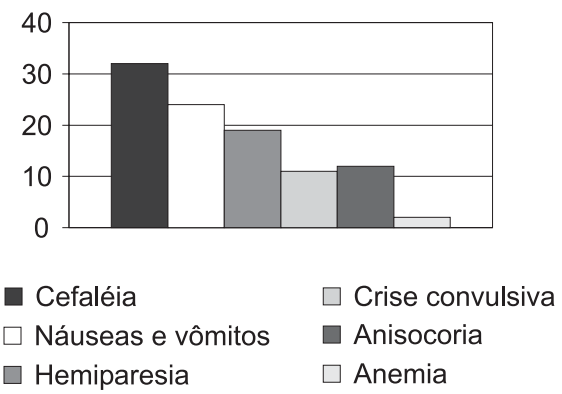

Gráfico 4 - Quadro neurológico.

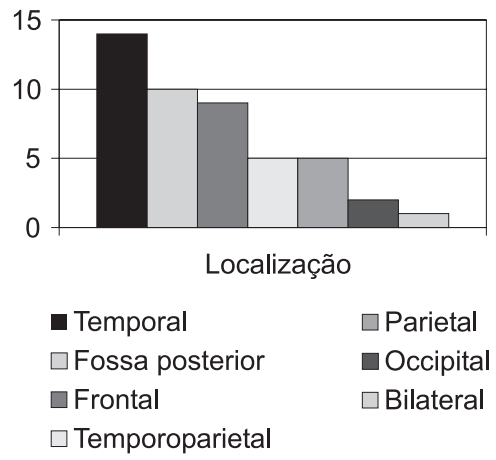

Gráfico 5 - Localização.

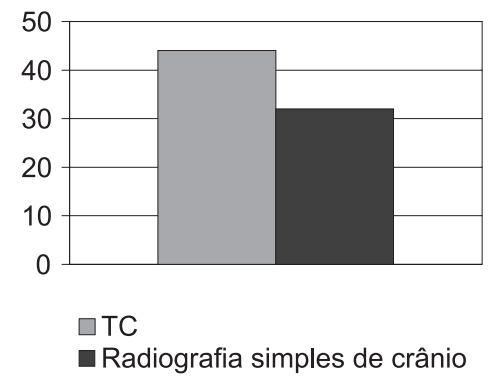

Gráfico 6 - Exames complementares positivos. 

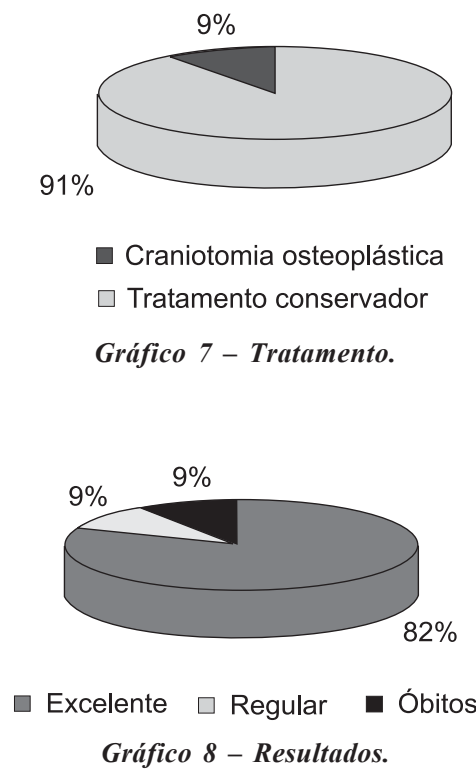

\section{Discussão}

O traumatismo craniano é comum em crianças. É responsável por aproximadamente 600 mil visitas anuais aos departamentos de emergência nos EUA. Alguns desses são traumas leves. O principal objetivo do profissional na emergência é identificar e tratar algumas das lesões cerebrais que põem em risco a vida. Em virtude do uso da TC nos serviços de emergência, a detecção precoce de complicações secundárias do traumatismo craniano são identificadas prontamente e tratadas adequadamente; com isso, tem-se diminuído a morbimortalidade nesses pacientes.

O HED é raro, não ocorrendo em mais que 4\% dos pacientes hospitalizados com traumatismo craniano $^{10,25,34}$. O HED é pouco freqüente na infância em razão das peculiaridades anatômicas e fisiológicas nessa faixa etária, como a maior maleabilidade e elasticidade craniana; o sulco que aloja a artéria meningéia média é raso e, assim, durante o traumatismo, essa artéria se desloca sem sofrer lesão; a maior aderência da dura-máter ao crânio e a díploe craniana pouco proeminente diminui a possibilidade do desenvolvimento de um HED $^{14,21,22}$.

$\mathrm{Na}$ infância, as causas do HED são, geralmente, diferentes das verificadas em outras faixas etárias. $\mathrm{Na}$ maioria das vezes é em razão de traumatismo craniano por queda de altura, seguida de acidentes de trânsito, sendo mais raro pós-derivação ventriculoperitoneal, traumatismo do parto, discrasias sangüíneas e, em alguns casos, não se identifica a causa, levando, às vezes, a se pensar em mau-trato ${ }^{1,2,26,32,35,36}$. Em nosso trabalho, a queda acidental e os acidentes automobilísticos foram as principais causas, o que está de acordo com os achados da literatura médica.

O HED localiza-se quase sempre sobre a convexidade do hemisfério cerebral na fossa média. O HED bilateral é raro na infância. O HED da fossa posterior na infância ocorre entre $9 \%$ e $20 \%$ dos casos. Em nossa casuística, a localização temporal foi a mais freqüente, ocorrendo uma alta incidência de HED na fossa posterior - 10 casos $(22 \%)$-, fato este também observado por Beni-Adani e cols. ${ }^{5}$, que encontraram 4 casos nessa localização em 11 pacientes $(36,6 \%)$.

O quadro clínico do HED na infância é geralmente atípico $^{15,29,30,37}$. Algumas crianças apresentam-se ao hospital após um traumatismo relativamente leve ${ }^{35}$. Vários trabalhos têm demonstrado que lesões intracranianas sérias podem ocorrer após traumatismo craniano leve em pacientes alertas. Estudos realizados em adultos e crianças encontraram a incidência de $1 \%$ a $3 \%$ de hemorragia intracraniana em pacientes que se encontravam alertas após traumatismo craniano leve e moderado ${ }^{23}$. Cook e cols. ${ }^{11}$ relataram que em 100 pacientes com HED e escore de 14 ou 15 na Escala de Coma de Glasgow 40\% tinha náuseas ou vômitos, mas nenhum deficit neurológico focal. O quadro clínico clássico de perda da consciência após o traumatismo, acompanhado por um intervalo lúcido, seguido de midríase homolateral ao hematoma e hemiparesia contralateral, com diminuição do nível de consciência, ocorre em apenas $20 \%$ dos casos. A midríase unilateral é identificada em aproximadamente $40 \%$ dos casos (em nosso trabalho foi observado em $45 \%$ dos casos). Traumatismos que cursam com hipotensão arterial sistêmica ou hipertensão intracraniana alteram, de maneira significativa, a evolução clínica do $\mathrm{HED}^{8,24}$. Em crianças menores, o HED pode ser volumoso, podendo ocasionar um quadro de anemia aguda e com pouca sintomatologia neurológica, em razão de boa tolerância cerebral permitida pela elasticidade craniana e as fontanelas abertas ${ }^{15,30}$, fato este observado em dois dos nossos pacientes (Figura 1). Nos lactentes, predominam irritabilidade, choro fácil ou recusa alimentar, sendo o vômito, em todas as faixas etárias, um sintoma freqüente $^{19}$. A presença de sinais de acometimento cerebelar, com rigidez de nuca, nistagmo, paresia de nervos oculomotores e sonolência, juntamente com um traço de fratura do osso occipital, ou mesmo a presença do sinal de Battle, deve levantar a suspeita de um HED na fossa posterior ${ }^{3,12,31}$. Em nossa casuística, houve uma incidência alta de HED na fossa posterior - cerca de $22 \%$ dos casos -, e apenas um caso de localização bilateral supratentorial.

Em crianças pequenas, com freqüência, não se observam traços de fratura em razão das propriedades elásticas da abóbada craniana ${ }^{9,13,26,29,37}$, porém, em 


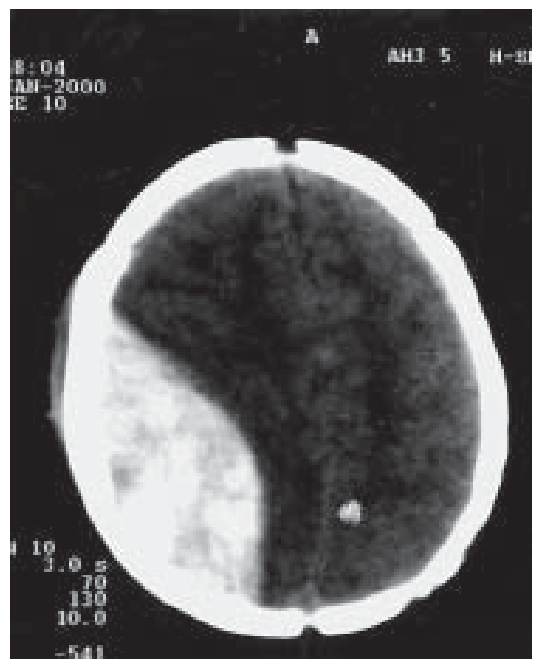

Figura 1 - TC demonstrando extenso hematoma extradural parietoccipital direito em uma criança com 4 meses de idade, que apresentou anemia.

crianças maiores, tem sido relatada uma incidência de traço de fratura craniana que varia de $60 \%$ a $80 \%$ dos $\operatorname{casos}^{10,15,16,21}$. Em nosso trabalho, encontramos uma incidência de $73 \%$ dos casos. Portanto, um exame de raios $\mathrm{X}$ simples de crânio normal não exclui a presença de um HED na infância ${ }^{35}$.

A TC é o método de escolha para o diagnóstico: demonstra uma lesão extra-axial de forma biconvexa e hiperdensa, localizada geralmente na região temporal ou temporoparietal ${ }^{40}$; em algumas situações, mostra desvio das estruturas da linha média e lesões intradurais associadas (Figura 2).

Uma vez diagnosticado o HED, o tratamento é cirúrgico, por meio de craniotomia osteoplástica, se-

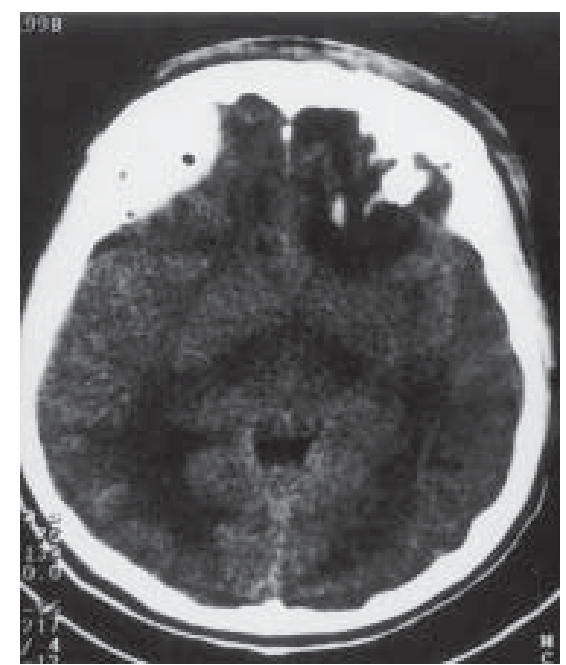

Figura 2 - TC apresentando hematoma extradural frontal direito e lesão intradural contralateral associada. guida da drenagem do hematoma e coagulação do vaso lesado $^{10,18}$. Em casos de HED na fossa posterior, está indicada craniectomia suboccipital unilateral ou mediana e drenagem do hematoma ${ }^{18,33}$. O tratamento conservador do HED tem sido realizado em casos com preservação do nível de consciência e na ausência de lesão intradural associada ${ }^{28}$. Para Knucley e cols. ${ }^{18}$, o tratamento conservador está indicado em pacientes assintomáticos desde que a TC realizada após seis horas ou mais do traumatismo demonstre um HED pequeno. Pozzati e cols. ${ }^{33}$ não recomendam o tratamento conservador, tendo em vista que pode ocorrer ressangramento a partir da cápsula do hematoma, como ocorre nos casos de hematoma subdural crônico. Em nossa casuística, 4 pacientes foram submetidos a tratamento conservador em razão de sua localização (frontal: 2 casos; parietal: 1 caso; e occipital: 1 caso), tamanho (menor que $2 \mathrm{~mm}$ ) em paciente alerta, com evolução satisfatória.

Em casos de HED na fossa posterior, está indicada prontamente a drenagem do hematoma, mesmo quando pequeno, pois pode ocasionar efeito de massa considerável em um espaço relativamente pequeno, que é a fossa posterior ${ }^{3,26}$. Wong e $\mathrm{Kwok}^{39}$ têm feito tratamento conservador em HED na fossa posterior usando os mesmos critérios para o HED supratentorial, com resultados excelentes, conduta esta realizada por outros autores ${ }^{6,28,33}$. A resolução espontânea do HED tem sido relatada de maneira esporádica na literatura médica ${ }^{38}$.

$\mathrm{O}$ tratamento cirúrgico em crianças com idades abaixo de 10 anos apresenta resultados satisfatórios ${ }^{15,17,26,29}$. Fatores que parecem ter grande influência no resultado são estado neurológico no momento da cirurgia, presença de lesão intradural associada, tempo decorrido entre o trauma e o início dos sintomas, tamanho e localização do hematoma ${ }^{4,7,16,20,29,34}$.

A mortalidade do HED na infância varia de $0 \%$ a $17 \%$ 7,10,14,19,21,29,37. Em nosso trabalho, a mortalidade observada foi de $9 \%$ (4 casos), sendo que 3 desses casos apresentavam lesões intradurais associadas.

O tratamento do HED na infância é considerado uma das intervenções neurocirúrgicas mais gratificantes para o neurocirurgião, já que, tratada precoce e adequadamente, a criança passa de um estado neurológico grave para um normal.

\section{Referências}

1. AGUIAR PH, SHU EBS, FREITAS ABR, LEME RJA, MIURA FK, MARINO Jr R: Causes and treatment of intracranial haemorrhage complicating shunting for paediatric hydrocephalus. Childs Nerv Syst 16:21821, 2000. 
2. AMMIRATI M, TOMITA T: Epidural hematomas in infancy and childhood. J Pediatr Neuroscience 1:1238, 1985.

3. ARKINS TJ, McLENNAN JE, WINSTON KR, STRAND RD, SUZUKI Y: Acute posterior fossa epidural hematomas in children. Am J Dis Child 131:690-2, 1977.

4. BEJJANI GK, DONAHUE DJ, RUSIN J, BROEMELING LD: Radiological and clinical criteria for the management of epidural hematomas in children. Pediatr Neurosurg 25:302-8, 1996.

5. BENI-ADANI L, FLORES I, SPEKTOR S, UMANSKY F, CONSTANTINI S: Epidural hematoma in infants: A different entity? J Trauma 46:306-11, 1999.

6. BOITEN J: Epidural haematoma of the posterior fossa: good results after prompt diagnosis with CT. J Neurol Neurosurg Psychiatr 52:914-5, 1989.

7. BRICOLO AP, PASUT LM: Extradural haematoma: toward zero mortality. Neurosurgery 14:8-12, 1984.

8. BUCCI MN, PHILLIPS TW, McGILLIANDY JE: Delayed epidural hemorrhage in hipotensive mul-tiple trauma patients. Neurosurgery 19:65-8, 1986.

9. CARTER AE: Extradural hemorrhage in a child, without skull fracture, following minor trauma. $J$ Neurosurg 17:155-6, 1960.

10. CHOUX M, GRISOLI F, PERAGUT JC: Extradural hematoma in children. Child Brain 1:337-47, 1975.

11. COOK RJ, DORSCH NWC, FEARNSIDE MR, CHASELING R: Outcome prediction in extradural hematomas. Acta Neurochir (Wien) 95:90-4, 1988.

12. COSTA-CLARA JM, CLARAMUNT E, LEY L, LAFUENT $\mathrm{J}$ : Traumatic extradural hematomas of posterior fossa in children. Childs Nerv Syst 12:145-8, 1996

13. DAVIES KG, JAMJOOM AB, BURGES NA: Childhood extradural hematoma not always obvious. $\mathrm{Br} \mathrm{J}$ Clin Practice 44:420-1, 1990.

14. DHELLEMES ESP, JEJUANINE JP, CHRISTAENS JL, COMBELLES G: Traumatic extradural hematomas in infancy and childhood. Experience with 144 cases. J Neurosurg 62:681-4, 1985.

15. HAWKES CD, OGLE WS: Atypical features of epidural hematoma in infants, children and adolescents. J Neurosurg 19:971-80, 1962.

16. JAMJOOM A: The influence of concomitant intradural pathology on the presentation and outcome of patients with acute traumatic extradural haematoma. Acta Neurochir (Wien) 115:86-9, 1992.

17. JAMJOOM A, CUMMINS B, JAMJJOOM ZA: Clinical characteristics of traumatic extradural hematoma: a comparison between children and adults. Neurosurgery 17:277-81, 1994.

18. KNUCKEY NW, GALBARD ST, EPSTEIN MH: The management of "asymptomatic" epidural hematomas. J Neurosurg 70:392-6, 1989.

19. LEGGATE JRS, LOPEZ-RAMOS N, GENITORI L, LENA G, CHOUX M: Extradural haematoma in infant. $\mathrm{Br} J$ Neurosurg 3:533-40, 1989.

20. LOBATO RD, RIVAS JJ, CORDOBÉS F, AETED E, PEREZ C, SARAIBA R et al.: Acute epidural hematoma. An analysis of factors influencing the outcome of patients undergoing surgery in coma. J Neurosurg 68:4857, 1988.

21. MAZZA C, PASQUALIN A, FERRIOTTI G, Da PIAN R: Traumatic extradural hematomas in children: experience with 62 cases. Acta Neurochir (Wien) 65:6780, 1982

22. MERRY GS, STUART G: Extradural hematoma in the neonate. Case report. J Neurosurg 51:713-4, 1979.

23. MILLER JD, MURRAY LS, TEASDALE GM: Development of a traumatic intracranial hematoma after a "minor" head injury. Neurosurgery 27:669-73, 1990.
24. MILO R, RAZON N, SCHIFFER J: Delayed epidural hematoma. A review. Acta Neurochir (Wien) 84:13-23, 1987.

25. MILZA PG, NARDI PV, GIGLI R, La MOTTA A: Epidural hematomas in infancy and childhood: report on 176 cases. J Pediatr Neurosci 5:117-22, 1989.

26. MOHANTY A, KALLURI VRS, SUBBAKRISHNA DK, SATISH S, CHANDRA MOULI BA, DAS BS: Prognosis of extradural haematomas in children. Pediatr Neurosurg 23:57-63, 1995.

27. MOLLOY CJ, McCAUL KA, McLEAN AJ, NORTH JB, SIMPSON DA: Extradural haemorrhage in infancy and childhood. A review of 35 years experience in South Australia. Childs Nerv Syst 6:383-7, 1990.

28. PANG D, HORTON J, HERRON JM, WILBERGER JE, VRIES JK: Nonsurgical management of extradural hematomas in children. J Neurosurg 59:958-71, 1983.

29. PASAOGLU A, ORTHON C, KOÇ K, SELÇUKLU A, AKDEMIR H, UZUMOGEU H: Traumatic extradural hematomas in pediatric age group. Acta Neurochir (Wien) 106:136-9, 1990.

30. PEREIRA CU, GENITORI L: Hematoma extradural no récem-nascido. Pediatria Moderna XXIX:253$5,1993$.

31. PEREIRA CU, BARRETO AS, SILVA AD, LEÃO JDBC, CARVALHO AF, PEREIRA FA: Hematoma extradural da fossa posterior. Considerações sobre 6 casos. Arq Bras Neurocir 13:168-72, 1994.

32. PEREIRA CU, PORTO MWS, HOLANDA RR, ANDRADE WT: Epidural hematoma after ventriculoperitoneal shunt surgery. Arq Neuro Psiquiatr 56:62932, 1998.

33. POZZATI E, TOGNETTI F, CARVALHO M, ACCIARI N: Extradural hematomas of the posterior cranial fossa: observations on a serie of 32 consecutive cases treated after the introduction of computed tomographic scanning. Surg Neurol 32:300-3, 1989.

34. RIVAS JJ, LOBATO RD, SARAIBA R, CORDOBÉS F, CABRERA A, GOMEZ P: Extradural hematoma: Analysis of factors influencing the courses of 161 patients. Neurosurgery 23:44-51, 1988.

35. SCHUTZMAN SA, BARNES PD, MANTELLO M, SCOTT RM: Epidural hematomas in children. Ann Emerg Med 22:535-41, 1993.

36. SHUGERMAN RP, PAEZ A, GROSSMAN DC, FELDMAN KW, GRADY MS: Epidural hemorrhage. It is abuse? Pediatrics 97:664-8, 1996.

37. SINGOUNAS EG, VOLIKAS ZG: Epidural haematoma in a pediatric population. Childs Brain 11:250-4, 1984

38. WEAVER D, POBERESKIN L, JANE JÁ: Spontaneous resolution of epidural hematomas. Report of two cases. J Neurosurg 54:248-51, 1981.

39. WONG CW, KWOK CK: Posterior fossa epidural haematoma in children. Asian J Surg 14:64-70, 1991.

40. ZIMMERMAN RA, BILANIUK LT: Computed tomographic staging of traumatic epidural bleeding. Radiology 144:809-12, 1982.

Original recebido em junho de 2001

Aceito para publicação em agosto de 2001

\section{Endereço para correspondência}

Prof. Dr. Carlos Umberto Pereira

Av. Augusto Maynard, 245/404

CEP 49015-380 - Aracaju, SE

E-mail:umberto@infonet.com.br 